\title{
Preface: Orthogonal Polynomials, Special Functions and Applications
}

\author{
By Peter A. Clarkson ${ }^{1}$ and Adri B. Olde Daalhuis ${ }^{2}$
}

The 14th International Symposium on "Orthogonal Polynomials, Special Functions and Applications" (OPSFA) took place at the University of Kent in Canterbury, UK during the week 3rd-7th July 2017, organised by the School of Mathematics, Statistics and Actuarial Science. The conference is the 14th event in the OPSFA series, with the first meeting held in Bar-Le-Duc, France in 1984, and this was the first one to take place in the UK. Over 150 participants from 22 countries took a pilgrimage to the city of Canterbury to tell a tale or to listen to one of the many interesting tales by the plenary lecturers and the contributed speakers. There were a diverse collection of plenary talks reflecting recent activity in the field, with 5 of the 12 plenary speakers women. Orthogonal polynomials were still prominent, with Chebyshev polynomials, exceptional orthogonal polynomials, Jacobi matrices, multivariate orthogonal polynomials and combinatorics of Koornwinder polynomials but other special functions also made their appearance. In particular Bessel functions, Painlevé equations, the Riemann zeta function, and Airy functions. Most notably there was a lot of emphasis on applications, more so than previous OPSFA meetings: approximation on the real line, cubature on regular polygons, random matrix theory, geometry of Painlevé equations, non-smooth waves, point processes and numerical methods.

A public lecture "Math is in the eye of the beholder" was given by Andrei Martínez Finkelshtein (Universidad de Almería, Spain) who presented a very entertaining insight into the mathematics of the eye, in particular medical diagnostic analysis and medical imaging of the cornea. For example Andrei explained that lenses of a shark eye are the best for replacing lenses in our eyes.

The Gábor Szegő prize was awarded to Thomas Trogdon (University of California, Irvine, USA), gave the lecture "The high oscillation of special functions", for his versatility in combining orthogonal polynomials and

Address for correspondence: School of Mathematics, Statistics \& Actuarial Science University of Kent, Canterbury, CT2 7FS, UK P.A.Clarkson@kent.ac.uk 


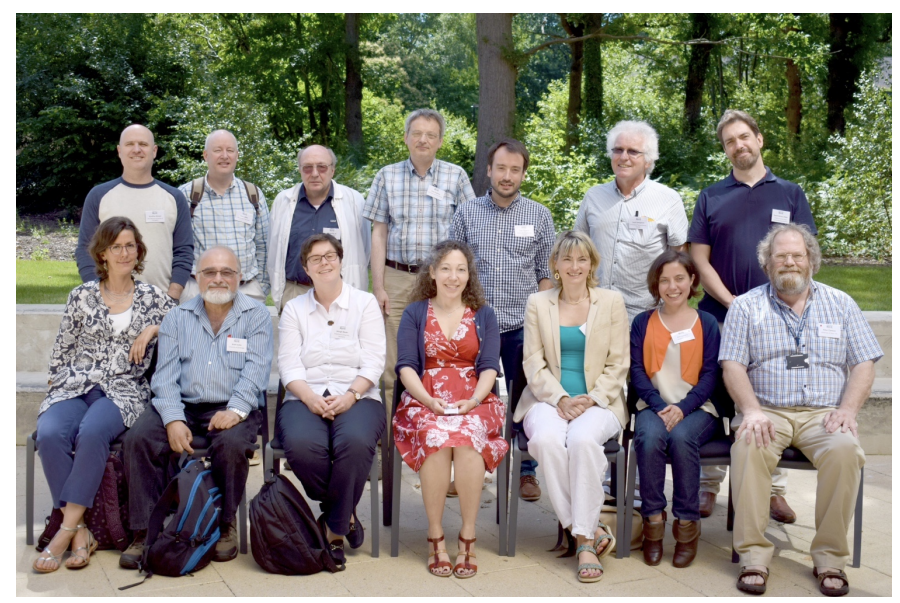

Figure 1. OPSFA14 plenary speakers and Gábor Szegő prize winner. Back row: Jonathan Breuer, Peter Miller, Alexander Its, Arno Kuijlaars, Tom Trogdon, Jacek Szmigielski, David Gómez-Ullate; Front row: Evelyne Hubert, Arieh Iserles, Margit Rösler, Nina Snaith, Marta Mazzocco, Ana Loureiro (organiser), Peter Clarkson (organiser).

special functions in new and creative ways to deduce results in a variety of fields.

This special issue features papers by plenary speakers at OPSFA14. These reflect the varied nature of talks at the conference with papers discussing aspects of Askey-Wilson polynomials [1], Sonine formulas for Bessel functions [2], cubatures [3], exceptional orthogonal polynomials [4, 5], rational solutions of Painlevé equations [6], and multipeakon solutions of partial differential equations [7].

Koornwinder and Mazzocco [1] investigate the degeneration of the Askey-Wilson polynomials, which are a four-parameter family of orthogonal symmetric Laurent polynomials that are eigenfunctions of a secondorder $q$-difference operator and of a second-order difference operator.

Rösler and Voit [2] study the extension of Sonine formulas for Bessel functions on symmetric cones which is achieved by an analytic extension of the involved beta measures as tempered distributions.

Collowald and Hubert [3] give a symbolic computation algorithm to characterize all cubatures, which are generalizations of quadratures to numerically compute multiple integrals.

Bonneux and Kuijlaars [4] discuss the asymptotic behavior of the regular zeros and the exceptional zeros of exceptional Laguerre polynomials 
as the degree tends to infinity by associating the polynomials with two partitions.

Gomez-Ullate, Grandati, and Milson [5] derive identities between determinants whose entries are Hermite polynomials which are applied to provide a more efficient representation for exceptional Hermite polynomials and for rational solutions of the fourth Painlevé equation.

Bothner, Miller, and Sheng [6] discuss rational solutions of the third Painlevé equation which generically depend on two parameters $m \in \mathbb{C}$ and $n \in \mathbb{Z}$, in particular in the limit of large $n$.

Anco, Chang, and Szmigielski [7] study multipeakon solutions of a family of $U(1)$-invariant partial differential equations which have a Hamiltonian structure.

\section{References}

1. Tom Koornwinder and Marta Mazzocco, Dualities in the $q$-Askey scheme and degenerated DAHA, Stud. Appl. Math., online DOI: 10.1111/sapm.12229.

2. Margit Rösler and Michael Voit, Beta distributions and Sonine integrals for Bessel functions on symmetric cones, Stud. Appl. Math., online DOI: 10.1111/sapm.12217.

3. Mathieu Collowald and Evelyne Hubert, Algorithms for computing cubatures based on moment theory, Stud. Appl. Math., online DOI: 10.1111/sapm.12xxx.

4. Niels Bonneux and Arno B.J. KuijlaArs, Exceptional Laguerre polynomials, Stud. Appl. Math., online DOI: 10.1111/sapm.12204.

5. David Gomez-Ullate, Yves Grandati, and Robert Milson, Durfee rectangles and pseudo-Wronskian equivalences for Hermite polynomials, Stud. Appl. Math., online DOI: 10.1111/sapm.12225.

6. Thomas Bothner, Peter D. Miller, and Yue Sheng, Rational solutions of the Painlevé-III equation, Stud. Appl. Math., online DOI: 10.1111/sapm.12220.

7. Stephen C. Anco, Xiangke Chang, and Jacek Szmigielski, The dynamics of conservative peakons in a family of $U(1)$-invariant integrable equations of NLSHirota type, Stud. Appl. Math., online DOI: 10.1111/sapm.12241.

${ }^{1}$ School of Mathematics, Statistics \& Actuarial Science, University of Kent, Canterbury, CT2 7FS, UK

${ }^{2}$ Maxwell Institute and School of Mathematics, The University of Edinburgh, Peter Guthrie Tait Road, Edinburgh EH9 3FD, UK 